\title{
Prostate cancer volume estimations based on transrectal ultrasonography-guided biopsy in order to predict clinically significant prostate cancer
}

\author{
Ersin Konyalioglu ${ }^{1}$, Hüseyin Tarhan ${ }^{2}$, Ozgur Cakmak ${ }^{2}$, Emel Ebru Pala ${ }^{3}$, Ferruh Zorlu ${ }^{2}$ \\ ${ }^{1}$ Department of Urology, Milas 75. Yil State Hospital, Mugla, Turkey; ${ }^{2}$ Department of Urology, Tepecik \\ Research and Training Hospital, Izmir, Turkey; ${ }^{3}$ Department of Pathology, Tepecik Research and Training \\ Hospital, Izmir, Turkey
}

\section{ABSTRACT}

Introduction: Tumor diameter is a reliable parameter to estimate tumor volume in solid organ cancers; its use in prostate cancer is controversial since it exhibits a more irregular pattern of growth. This study aimed to examine the association between the tumor volume estimations based on transrectal ultrasound (TRUS) guided biopsy results and the tumor volume measured on the pathological specimen.

Materials and Methods: A total of 237 patients who underwent radical retropubic prostatectomy (RRP) were included in this retrospective study. The differences and correlations between cancer volume estimations based on TRUS guided biopsy findings and cancer volume estimations based on post-prostatectomy pathology specimens were examined. In addition, diagnostic value of TRUS guided biopsy-based volume estimations in order to predict clinically significant cancer ( $>0.5 \mathrm{cc}$ ) were calculated. Results: The mean cancer volume estimated using TRUS biopsy results was lower $(5.5 \pm 6.5 \mathrm{cc})$ than the mean cancer volume calculated using prostatectomy specimens $(6.4 \pm 7.6 \mathrm{cc})(\mathrm{p}<0.041)$. TRUS guided biopsy examination resulted in 5 false positive and 15 false negative cases. There was a significant but weak correlation between the two parameters $(\mathrm{r}=0.62, \mathrm{p}<0.001)$. The sensitivity and specificity of TRUS guided biopsy in predicting the presence of clinically significant cancer was 93.4\% (95\% CI, 89.1-96.1) and 50.0\% (95\% CI, 20.1-79.9), respectively.

Conclusions: TRUS guided biopsy-derived estimations seem to have a limited value to predict pathologically established tumor volume. Further studies are warranted to identify additional methods that may more accurately predict actual pathological characteristics and prognosis of prostate cancer.

\section{ARTICLE INFO}

\section{Key words:}

Ultrasound, High-Intensity

Focused, Transrectal; Prostatic

Neoplasms; Tumor Burden

Int Braz J Urol. 2015; 41: 442-8

Submitted for publication:

May 22, 2014

Accepted after revision:

October 19, 2014

\section{INTRODUCTION}

Radical prostatectomy is currently the treatment of choice for prostate cancer in patients with a good expectancy of life. However, an active surveillance protocol rather than curative tre- atment is recommended in organ-confined disease for low to very-low risk tumors and for "clinically insignificant" tumors according to Epstein's criteria. On the other hand, tumors with a volume of less than $0.5 \mathrm{cc}$ are also considered "clinically insignificant" (1). But it should be also stated that 
"insignificant cancer" in surgical specimens is not only a limited volume cancer but should be also confined to the organ and with Gleason score $3+3=6$.

Maximum tumor diameter is a reliable parameter to estimate tumor volume in solid organ cancers, which generally have a roughly spherical form. However, this may not be readily applied to prostate cancers, which are thought to exhibit a more irregular pattern of growth (2). Therefore, the utility of this approach is controversial for prostate cancer despite the presence of a consensus regarding the role of tumor size as a staging parameter for many different solid tumors.

In the recent consensus about reports on radical prostatectomy specimen, the ISUP members decided that pathologist should give some estimative of tumor volume but its importance is still under investigation. Several different methods such as square, cuboid, or ellipsoidal estimations have been utilized to gauge the tumor volume of radical prostatectomy specimens (3); and these approaches require the use of 3-dimensional measurements (length, width, and thickness) for tumor volume estimation that cannot be accomplished through transrectal ultrasound (TRUS) guided biopsy. Therefore, these methods cannot be used to estimate the tumor volume of the biopsy.

In the present study it was assessed the tumor volume estimation prior to radical prostatectomy, the association between the tumor volume estimate based on TRUS biopsy results, the tumor volume measured on the pathological specimen, and the value of this method to predict the presence of clinically significant cancer.

\section{MATERIALS AND METHODS}

\section{Patients}

A total of 318 patients who underwent radical retropubic prostatectomy (RRP) in our urology department between March 2006 and June 2012 were retrospectively examined. For biopsy standardization, only patients with 10 and 12-quadrant biopsy results with a minimum core length of $11.5 \mathrm{~mm}$ were included in the study. Twenty-nine patients without a 10 or 12-quadrant biopsy result, 14 patients with an average core length below $11.5 \mathrm{~mm}$, and 38 patients with missing data were excluded, yielding a total of 237 subjects in the study population.

\section{Assessments}

Cancer volume estimations based on TRUS guided biopsy findings and cancer volume estimations based on post-prostatectomy pathology specimens were assessed.

Prostate biopsies were performed by a trained team with the patient in lateral decubitus position. Periprostatic nerve block was performed in each patient using prilocaine. In all patients, a SonoScape ${ }^{\circledR}$ SSI 2000 BW US biopsy gun with a $6.5 \mathrm{MHz}$ transrectal probe and an $18 \mathrm{G}, 25 \mathrm{~cm}$ Geotek ${ }^{\circledR}$ biopsy needle were used. In case of inadequate specimen collection, the procedure was repeated. Each core was sent to the pathology laboratory with the name of the patient; localization data were recorded on the sample container, and all biopsies were assessed by the same pathology laboratory. In each cancer-positive core, the maximum tumor dimension was reported by the pathology department. Pathology specimens were analyzed by five different pathologists, one of whom experienced in uropathology. In core biopsies we measured each tumor focus individually and substracted out the intervening benign prostate tissue. The sum of these measurements was considered the cancer length/diameter of the core. Then we calculated the approximate tumor volume by $4 / 3 . \pi \cdot r^{3}$ formula which is actually used for calculating the sphere volume. For each cancer-positive focus, the cancer volume based on this formula was calculated separately. The resultant data were collected and recorded as the estimated cancer volume for the TRUS-biopsy. In the literature there is no previous study using this formula for estimating the cancer volume in biopsy specimen. However, we know that one of the most reliable method to measure total prostate volume is spherical formula, so we tried this empirical method as a new approach.

In radical prostatectomy specimens, firstly the percentage of tissue involved with tumor was evaluated by simple visual inspection. In possible cases we measured the dominant tumor nodule in 
at least 2 dimensions. For the measurement of approximate cancer volume in radical prostatectomy specimens, the entire prostate was examined. We calculated the maximal area and percentage of the tumor in tumor involved blocks and determined the ratio of involved-to-uninvolved blocks. We estimated a percentage of prostate involved by prostate cancer. And we calculated the total prostate volume by using three dimensions. We found out the tumor volume by using total prostate volume and tumor percentage. We did not use morphometric techniques.

Cancer volume greater than $0.5 \mathrm{cc}$ was considered significant cancer.

\section{Statistical analysis}

Analysis of data was done using Statistical Package for Social Sciences (SPSS) for Windows version 21. The difference between the cancer volume estimates based on TRUS guided biopsy findings and the cancer volume estimates in pathological specimens was compared using student $\mathrm{t}$ test for paired samples. The correlation between the two variables was tested using Spearman's correlation analysis. Diagnostic parameters including sensitivity, specificity, positive predictive value, negative predictive value and likelihood ratio of TRUS guided biopsy based volume estimations to predict clinically significant cancer were calculated. Receiver operator characteristic curve (ROC) was generated to examine the accuracy of TRUS-based estimations and potential cut-off values. A $p$ value $<0.05$ indicated statistical significance.

\section{RESULTS}

The mean age of the patients at the time of TRUS examination was 63.1 \pm 6.4 years (range, 44-77 y). At baseline, the mean PSA level was $10.6 \mathrm{ng} / \mathrm{mL}$. On TRUS the mean core length was $14.1 \mathrm{~mm}$ and mean prostate volume was $43.8 \mathrm{cc}$. The mean percentage of cancer in each core was as follows: right lateral 1, 12.7\%; right lateral 2, $11.0 \%$; right lateral 3, 9.8\%; right apex, $13.7 \%$; right middle, $12.6 \%$; right base, $11.3 \%$; left lateral 1 , 10.2\%; left lateral 2, 9.8\%; left lateral 3, 7.2\%; left apex, 9.7\%; left middle, 11.8\%; left base, 10.2\%.
In comparison, the mean percentage of cancer was $14.7 \%$ in prostatectomy specimens.

\section{COMPARISON OF CANCER VOLUMES}

The mean cancer volume estimated using TRUS biopsy results was $5.5 \pm 6.5$ cc (range, 0.21 $42.0 \mathrm{cc}$ ), while the mean cancer volume calculated using prostatectomy specimens was $6.4 \pm 7.6$ cc (range, 0-48.4 cc). The difference between the two mean volumes was statistically significant $(p<0.041)$. There was a significant but weak correlation between the two parameters $(r=0.62$, $\mathrm{p}<0.001)$.

\section{PREDICTIVE VALUE OF TRUS GUIDED BIOPSY FINDINGS}

The sensitivity and specificity of TRUS guided biopsy in predicting the presence of clinically significant cancer was 93.4\% (95\% CI, 89.196.1) and 50.0\% (95\% CI, 20.1-79.9), respectively. Its positive predictive value was $97.7 \%$ and negative predictive value was $25 \%$, with a positive likelihood ratio of 3.5. In this study, TRUS guided biopsy examination resulted in 5 false positive and 15 false negative cases. Figure- 1 shows a receiver operator characteristics (ROC) curve for predicting clinically significant cancer based on TRUS guided biopsy estimation. Area under the curve is 0.81 (95\% CI, 0.65-0.96, $\mathrm{p}<0.001$ ) indicating a good to fair accuracy. A cut-off value $>0.41$ cc would result in a better sensitivity with the same level of specificity with 0.5 cc $(97 \%$ and $50 \%$, respectively), whereas when the cut-off is set to>0.74 cc, sensitivity would decrease and specificity would increase slightly (87\% and 60\%, respectively).

Tumors with a volume of less than $0.5 \mathrm{cc}$ are considered "clinically insignificant", tumors with a volume of more than $0.5 \mathrm{cc}$ are considered "clinically significant". In our study TRUS guided biopsy examination resulted in 5 false positive and 15 false negative cases. Fifteen patients with clinically non-significant lesion according to tumor volume in TRUS guided biopsy were subsequently found to have a clinically significant lesion after RRP. In contrast, 5 patients having clinically significant lesion according to TRUS 
Figure 1 - ROC curve for predicting clinically significant cancer, based on TRUS guided biopsy estimation.

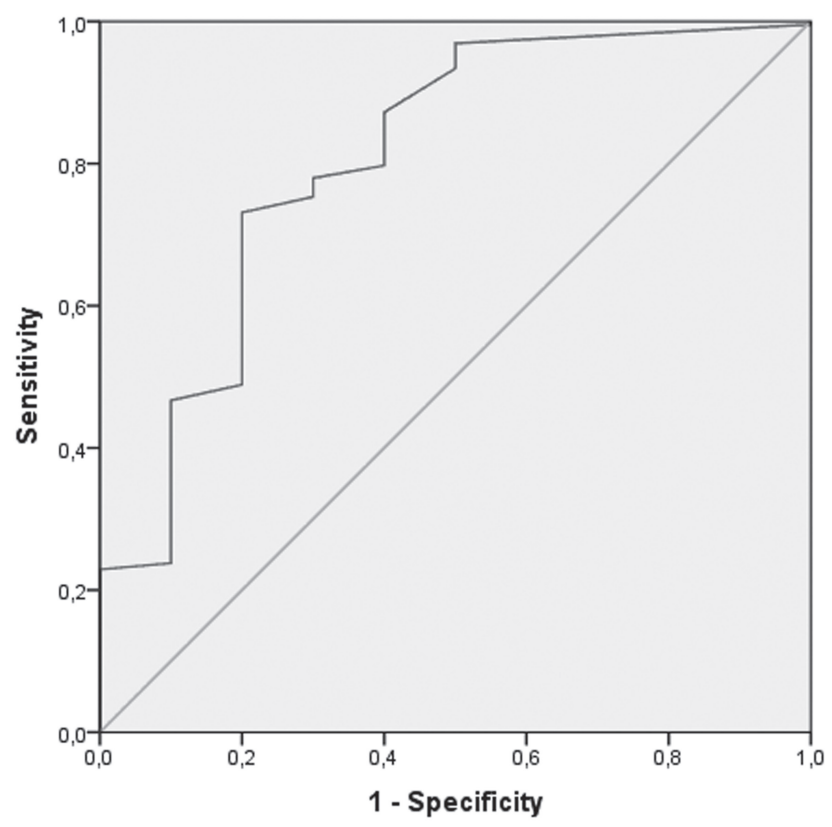

guided biopsy were found to have an insignificant tumor after RRP.

\section{DISCUSSION}

TNM classification and the Gleason scoring system, i.e. histological subtyping, provide a fairly high predictive value in prostate cancer (2). Many other solid organ cancers lack parameters with such a high predictive value for disease progression, placing a significant importance on the tumor volume estimations.

Presence of tumor in at most 3 quadrants with no>50\% tumor in any of the cores is one of the Epstein's criteria, which aim to define "clinically non-significant" cancers that do not require curative treatment (4). However, if prostate cancer has an irregular pattern of growth as suggested by some authors (2), then how appropriate would it be to classify a cancer as "clinically non-significant" solely on the basis of the number of cancer-positive biopsy cores and the percentage of tumor in the cores. Do cancers classified as "clinically non-significant" according to such criteria really have a volume of less than 0.5 cc? And finally, can the cancer volume be predicted on the basis of biopsy results and are these consistent with the cancer volume in RRP materials? These questions, and particularly the latter, were the primary questions that were addressed in our study.

The first study to draw attention to the importance of tumor volume in prostate cancer was carried out by Stamey et al. where tumor volume was found to be associated with metastases, seminal vesicular invasion, and extra-capsular involvement, with only those prostate cancers exceeding a volume of $4 \mathrm{cc}$ having association with metastases in 100 autopsy-prostates and 38 radical prostatectomy specimens (5).

In 1993, Epstein et al. examined 185 radical prostatectomy specimens and found no predictive value for the tumor volume, when Gleason score and surgical margins were incorporated into the assessment. Although tumors with a volume of greater than 10 cc showed progression and tumors smaller than $0.5 \mathrm{cc}$ showed no progression, tumor volume had no predictive value for tumors that lie in the intermediate volume category, i.e. between these two values, which comprise the majority of such tumors . A similar study from John Hopkins found no progression at all in tumors with a volume of less than $0.5 \mathrm{cc}$, while $97 \%$ of the tumors with a volume greater than 12 cc progressed. In recent publications, the reported rates of progression for tumor volumes of $0.5 \mathrm{cc}$ to $2 \mathrm{cc}$ and $2 \mathrm{cc}$ to 6 cc are $14 \%$ and 39\%, respectively (6).

In 1990, Humphrey and Vollmer examined 73 radical prostatectomy specimens and found that the percent-tumor involvement of the specimen had an independent predictive value for clinical recurrence (7). The percent-tumor involvement in progressing and non-progressing tumors in the studies by Carvalhal et al. and Jones et al. were $23.8 \%$ and 11.3 , and $36 \%$ and $24 \%$, respectively $(8,9)$. The percentage of tumor involvement in our study was $14.7 \%$, which was in line with previous reports, although no differentiation between progressing and non-progressing tumors was made in our study.

The most likely explanation for the discordant results relating to the independent prognostic significance of RP tumor volume lies on its strong correlation with other prognostic markers such as 
extraprostatic extension (EPE) and positive margins. In almost all studies RP tumor volume, EPE and margin status all correlated strongly with postoperative progression on univariate analysis. However, on multivariate analysis often one of these parameters drops out and loses its prognostic significance, as these 3 variables were tightly correlated with each other. For example, once margin status is known, tumor volume (TV) does not provide much additional prognostic information and vice versa. Consequently, in a given study, depending on whether TV or margin status is slightly better, one variable will be highly significant on multivariate analysis and the other will loose its significance. For example, if in one study margins were not factored into the multivariable analysis, TV was strongly predictive at $\mathrm{p}=0.004$ (10). However, once margins were factored into the analysis, TV lost prognostic significance (2).

Carvalhal et al. reported an average prostate volume of $46 \mathrm{cc}$, while this figure reported by Jung et al. was 38 cc $(9,11)$. Obek et al. observed an average prostate volume of $39.80 \mathrm{cc}$ and 46.3 cc in malignant or benign prostates, respectively (12). The average prostate volume in our study, i.e. $43.8 \mathrm{cc}$, was consistent with literature data.

One of the most controversial aspects of TRUS biopsy reports is the absence of an agreement on the measurement methods for multiple cancer foci, which exhibit discontinuous areas of involvement with normal prostate tissue in-between. Pathologists' use of different reporting systems for discontinuous foci of cancer within the core may result in a variation in therapeutic options recommended. For instance, in the study by Egevad et al., a cancer without tumor continuity in the core was reported as having 60\% tumor involvement by one of the clinics, while in another clinic the normal prostate tissue between tumor involved sites were discarded, yielding a percent involvement below 50\% (13). One of the criteria for active surveillance is the absence of $>50 \%$ involvement in any core. In this situation, the recommendations would lead to different treatment schemes in the example above.

Pathologists in our hospital also discard benign prostate tissue between tumor-involved sites while determining percent tumor involvement.
Quintal et al. reported that total percent tumor involvement in biopsy specimens was superior to other volumetric measurement parameters in predicting $\mathrm{pT} 3$ in the multivariate analysis and represented an independent risk factor for biochemical recurrence when combined with PSA and Gleason score (14). These findings are somehow at odds with ours, since in our study, parameters utilized for estimating the biopsy tumor volume such as the tumor diameter or percent tumor involvement did not emerge as reliable predictive factors. The tumor volume estimates based on biopsy parameters were not consistent with the tumor volume observed in RRP materials and 15 patients were classified as clinically non-significant according to biopsy based tumor volume and actually had clinically significant tumors after RRP. We do believe that these observations may well be associated with the irregular growth pattern of the prostate cancer.

Boccon-Gibod et al. proposed a minimum core length of $10 \mathrm{~mm}$ for a good biopsy sampling (15). Similarly, Obek et al. found an increased cancer detection rate with a core length exceeded 11.9 $\mathrm{mm}$ compared to those below that cut-off value, i.e. cancer detection rates of $23 \%$ and $39 \%$, respectively (12). The average core length in biopsies with cancer detection was $12.3 \mathrm{~mm}$ vs. $11.4 \mathrm{~mm}$ in those without cancer detection ( $p=0.015)$. In our study, the average core length was $14.08 \mathrm{~mm}$, as suggested in previous reports. For biopsy standardization, 14 patients with an average core length below $11.5 \mathrm{~mm}$ were excluded from our analyses.

There is no literature data comparing biopsy based tumor volume estimations and surgical specimen tumor volume measurements. Perera et al. compared the tumor volume (TV) measurement methods in radical prostatectomy materials (3). TV estimation methods including square, cuboidal and elipsoidal approaches were compared against the 'gold standard' and the accuracy of identifying clinically significant tumors with a volume of $>0.5$ cc was determined. The elipsoidal method $(\mathrm{k}(\pi / 6)$ $\times$ length $\times$ width $\times$ thickness) produced the closest correlation with the gold-standard. However, these measurement methods require the use of 3-dimensional data obtained from the tumor tissue. In contrast, biopsy material provides only single-di- 
mensional data, precluding the use of these methods for estimating the tumor volume in biopsy materials. Kondler et al. used the ellipsoid formula to estimate TV in RP specimen and reported that increasing TV was associated with significantly higher risks of seminal vesicle invasion, positive surgical margins and lymph node involvement. Moreover, on multivariate analysis, greater TV remained associated with significantly increased risks of systemic progression, death from prostate cancer and all-cause mortality (16).

In their study, Ongun et al. reported that prostate specific antigen density (PSAD), maximum percentage in positive cores and radical prostatectomy (RP) tumor volume emerged as statistically significant determinants for advanced stage disease in their univariate and multivariate analyses. Also, ROC analyzes identified the radical prostatectomy tumor volume as a reliable parameter for advanced stage disease (17). As suggested by Ongun et al., ability to estimate the tumor volume prior to RP would help minimize treatment failures (i.e. over or undertreatment) in PCa. In our study, our objective was to estimate the tumor volume before RP. Our results showed that the average biopsy tumor volume was approximately $1 \mathrm{cc}$ less than the volume determined after pathological examination of the surgical specimen, the difference between the two being statistically significant $(\mathrm{p}<0.041)$. In addition, there was a weak correlation between cancer volumes predicted by TRUS biopsy and RRP specimen.

Tumors with a volume of less than $0.5 \mathrm{cc}$ are considered "clinically insignificant", tumors with a volume of more than $0.5 \mathrm{cc}$ are considered "clinically significant". In our study there were 5 false positive and 15 false negative results. We believe that this inconsistency between tumor volumes based on biopsy and surgery specimens may result from the irregular growth pattern of the prostate cancer.

\section{CONCLUSIONS}

Most of solid organ cancers grow spherically, though prostate cancer grows irregularly. So tumor diameter does not correlate with tumor volume as close as tumors in other sites. It is in- dicated that objective tumor volume measurement does not add useful prognostic information other than the information routinely reported by the pathologists. As the morphometric methods are not practical and time consuming, easy methods are used in routine practice. In this study we tried a new, easy method for volume estimation.

However, we could not find a strong correlation between tumor volume in biopsies and radical materials. Our data suggests that biopsy-derived estimation parameters currently available may have a limited value in the prediction of pathologically established tumor volume. Further studies are warranted to identify additional parameters and/or methods that may more accurately predict actual pathological characteristics and prognosis in prostate cancer.

\section{CONFLICT OF INTEREST}

None declared.

\section{REFERENCES}

1. Klein EA. What is 'insignificant' prostate carcinoma? Cancer. 2004;101:1923-5.

2. Epstein Jl. Prognostic significance of tumor volume in radical prostatectomy and needle biopsy specimens. J Urol. 2011;186:790-7.

3. Perera M, Lawrentschuk N, Bolton D, Clouston D: Comparison contemporary methods of estimating prostate tumor volume in pathological specimens. BJU Int 2013;113(Suppl 2):2934.

4. Epstein JI, Walsh PC, Carmichael M, Brendler CB. Pathologic and clinical findings to predict tumor extent of nonpalpable (stage T1c) prostate cancer.JAMA. 1994;271:368-74.

5. Stamey TA, McNeal JE, Yemoto CM, Sigal BM, Johnstone IM. Biological determinants of cancer progression in men with prostate cancer. JAMA. 1999;281:1395-400.

6. Carter HB, Sauvageot J, Walsh PC, Epstein JI. Prospective evaluation of men with stage T1C adenocarcinoma of the prostate. J Urol. 1997;157:2206-9.

7. Humphrey PA, Vollmer RT. Intraglandular tumor extent and prognosis in prostatic carcinoma: application of a grid method to prostatectomy specimens. Hum Pathol. 1990;21:799-804.

8. Carvalhal GF, Humphrey PA, Thorson P, Yan Y, Ramos CG, Catalona WJ. Visual estimate of the percentage of carcinoma is an independent predictor of prostate carcinoma recurrence after radical prostatectomy. Cancer. 2000;89:1308-14. 
9. Jones TD, Koch MO, Lin H, Cheng L. Visual estimation of tumour extent is not an independent predictor of prostate specific antigen recurrence. BJU Int. 2005;96:1253-7.

10. Dvorak T, Chen MH, Renshaw AA, Loffredo M, Richie JP, D'Amico AV. Maximal tumor diameter and the risk of PSA failure in men with specimen-confined prostate cancer. Urology. 2005;66:1024-8.

11. Jung JW, Lee BK, Choi WS, Park YH, Lee S, Jeong SJ, et al. Combination of clinical characteristics and transrectal ultrasound-guided biopsy to predict lobes without significant cancer: application in patient selection for hemiablative focal therapy. Prostate Int. 2014;2:37-42.

12. Öbek C, Doğanca T, Erdal S, Erdoğan S, Durak H. Core length in prostate biopsy: size matters. J Urol. 2012;187:2051-5.

13. Egevad L, Allsbrook WC Jr, Epstein Jl. Current practice of diagnosis and reporting of prostate cancer on needle biopsy among genitourinary pathologists. Hum Pathol. 2006;37:292-7.
14. Quintal MM, Meirelles LR, Freitas LL, Magna LA, Ferreira $\mathrm{U}$, Billis A. Various morphometric measurements of cancer extent on needle prostatic biopsies: which is predictive of pathologic stage and biochemical recurrence following radical prostatectomy? Int Urol Nephrol. 2011;43:697-705.

15. Boccon-Gibod L, van der Kwast TH, Montironi R, BocconGibod L, Bono A; European Society of Uropathology; European Society of Pathology Uropathology Working Group. Handling and pathology reporting of prostate biopsies. Eur Urol. 2004;46:177-81.

16. Knoedler JJ, Karnes RJ, Thompson RH, Rangel LJ, Bergstralh EJ, Boorjian SA. The association of tumor volume with mortality following radical prostatectomy. Prostate Cancer Prostatic Dis. 2014;17:144-8.

17. Ongun S, Celik S, Gül-Niflioglu G, Aslan G, Tuna B, Mungan U, et al. Are active surveillance criteria sufficient for predicting advanced stage prostate cancer patients? Actas Urol Esp. 2014;38:499-505.

Correspondence address: Ersin Konyalioglu, MD Department of Urology Milas 75. Yil State Hospital, Mugla, Turkey Fax: +90 252 513-5600 E-mail: konersin@yahoo.com 